\title{
Bioinformatics and Transcriptome Analysis of CFEM Proteins in Fusarium graminearum
}

\author{
Lingqiao Chen $1,2,+$, Haoyu Wang ${ }^{1,2,+}$, Junhua Yang ${ }^{2}$, Xianli Yang ${ }^{2}$, Mengyuan Zhang ${ }^{1,2}$, Zhihui Zhao ${ }^{2}$, \\ Yingying Fan ${ }^{3}$, Cheng Wang ${ }^{3}$ and Jianhua Wang $1,2, *$ (D) \\ 1 College of Food Science \& Technology, Shanghai Ocean University, Shanghai 201306, China; \\ m190300688@st.shou.edu.cn (L.C.); m200300781@st.shou.edu.cn (H.W.); m210300781@st.shou.edu.cn (M.Z.) \\ 2 Shanghai Key Laboratory of Protected Horticultural Technology, Laboratory of Quality and Safety Risk \\ Assessment for Agro-Products (Shanghai), Institute for Agro-Food Standards and Testing Technology, \\ Ministry of Agriculture, Shanghai Academy of Agricultural Sciences, 1000 Jinqi Road, \\ Shanghai 201403, China; yangjunhua@saas.sh.cn (J.Y.); yangxianli@saas.sh.cn (X.Y.); \\ 18918162068@163.com (Z.Z.) \\ 3 Institute of Quanlity Standards \& Testing Technology for Agro-Products, Xinjiang Academy of Agricultural \\ Sciences, Urumqi 830091, China; fyyxaas@xaas.ac.cn (Y.F.); wangcheng312@xaas.ac.cn (C.W.) \\ * Correspondence: wangjianhua@saas.sh.cn; Tel.: +86-2167131637 \\ + These authors contributed equally to this work.
}

check for updates

Citation: Chen, L.; Wang, H.; Yang, J.; Yang, X.; Zhang, M.; Zhao, Z.; Fan, Y.; Wang, C.; Wang, J. Bioinformatics and Transcriptome Analysis of CFEM Proteins in Fusarium graminearum. J. Fungi 2021, 7, 871. https://doi.org/ 10.3390/jof7100871

Academic Editor: Łukasz Stępień

Received: 26 September 2021

Accepted: 14 October 2021

Published: 16 October 2021

Publisher's Note: MDPI stays neutral with regard to jurisdictional claims in published maps and institutional affiliations.

Copyright: (c) 2021 by the authors. Licensee MDPI, Basel, Switzerland. This article is an open access article distributed under the terms and conditions of the Creative Commons Attribution (CC BY) license (https:// creativecommons.org/licenses/by/ $4.0 /)$.

\begin{abstract}
Fusarium blight of wheat is usually caused by Fusarium graminearum, and the pathogenic fungi will secrete effectors into the host plant tissue to affect its normal physiological process, so as to make it pathogenic. The CFEM (Common in Fungal Extracellular Membrane) protein domain is unique to fungi, but it is not found in all fungi. The CFEM protein contained in F. graminearum may be closely related to pathogenicity. In this study, 23 FgCFEM proteins were identified from the F. graminearum genome. Then, features of these proteins, such as signal peptide, subcellular localization, and transmembrane domains, etc., were analyzed and candidate effectors were screened out. Sequence alignment results revealed that each FgCFEM protein contains one CFEM domain. The amino acids of the CFEM domain are highly conserved and contain eight spaced cysteines, with the exception that FgCFEM8, 9, and 15 lack two cysteines and three cysteines were missed in FgCFEM18 and FgCFEM22. A recently identified CFEM_DR motif was detected in 11 FgCFEMs, and importantly we identified two new conserved motifs containing about 29 and 18 amino acids (CFEM_WR and CFEM_KF), respectively, in some of FgCFEM proteins. Transcriptome analysis of the genes encoding CFEM proteins indicated that all the CFEM-containing genes were expressed during wheat infection, with seven and six genes significantly up- and down-regulated, respectively, compared with in planta and in vitro. Based on the above analysis, FgCFEM11 and FgCFEM23 were predicted to be F. graminearum effectors. This study provides the basis for future functional analyses of CFEM proteins in F. graminearum.
\end{abstract}

Keywords: Fusarium graminearum; CFEM domain; candidate effectors; bioinformatics analysis; transcriptional analysis

\section{Introduction}

Fusarium graminearum species complex (FGSC) is an economically important plant pathogen causing Fusarium head blight (FHB) of wheat, barley, and other cereal crops worldwide [1,2]. FHB caused by FGSC is difficult to control and is known as the cancer of wheat. As one of the most destructive diseases worldwide, huge economic losses have been reported in Asia, Europe, North America, and many other countries [3]. FGSC has been ranked as the fourth most important plant pathogenic fungus [4]. In addition lowering grain yield, the disease mainly reduces grain quality, and results in mycotoxincontaminated grain. Fusarium strains can produce epoxy-sesquiterpenoid compounds 
known as trichothecenes, with deoxynivalenol (DON) predominated. These secondary metabolites are very stable and can enter the food chain with food processing which pose potent threat to human and animal health, and thus has aroused public safety concerns. Some trichothecenes can also act as virulence factors for pathogenicity in susceptible plant hosts, which can facilitate colonization and spreading of the fungi in host tissues [5-7]. The prevalence and widespread outbreaks of the devastating FHB disease, exacerbated by recent changes in climate and certain cropping practices, has posed a threat for global wheat production and food safety [8].

According to the interaction patterns with the hosts, plant pathogens are classified into biotrophic, necrotrophic, and hemibiotrophic pathogens. The economically important and destructive hemibiotrophs, such as FGSC and Magnaporthe oryzae, were believed to be typical hemibiotrophic fungi [9] starting from the suppression of the host immune system in a biotrophic phase with living cells and followed by a later necrotrophic phase during which the pathogens kill plant cells for nutrient acquisition. However, the underlying interaction mechanisms between pathogens and plant hosts remain incompletely understood, especially in the early infection stages. The identification of proteinaceous effectors in a number of Ascomycetes, including biotrophic, necrotrophic, and hemibiotrophic pathogens is a typical example. Most fungal effector proteins identified so far are relatively small in size (usually $<200$ amino acids) and contain a high percentage ( $2 \%$ to $20 \%$ ) of cysteine residues [10]. Thus, small secreted cysteine-rich proteins (SSCPs) are known to be a common source of fungal effectors that trigger resistance or susceptibility in specific host plants. Hence, identification and function characterization of SSCPs is of great importance for the understanding of the complex pathogenic process of the pathogens.

Until now, several cysteine-rich domains have been identified in fungal proteins, such as those present in hydrophobins and epidermal growth factors (EGF) [11]. The CFEM (common in several fungal extracellular membrane proteins) domain is a unique motif found in fungi and usually sharing $\sim 60$ amino acids (AA), which contains eight conserved cysteine residues $[12,13]$. CFEM domain is found primarily in glycosylphosphatidylinositol (GPI)-anchored cell-wall proteins, and present in one or more copies (normally with one copy) near the $\mathrm{N}$ terminus of proteins. Although the domains in several hydrophobins also have eight cysteine residues, the domain size, cysteine spacing and pattern of residues is completely distinct when compared with those of CFEM [12,14,15]. CFEM is similar to several EGF-like domains in domain size and pattern of cysteine residues, however, the cysteine residues do not align with any of the EGF-like domains. The features of CFEM suggest that it is a novel domain with characteristics distinct from the known cysteine-rich domains.

Many CFEM domain proteins have been found exclusively from Ascomycota and Basidiomycota, and they were found to be enriched in pathogenic fungi [13]. For example, there are 3, 8, 19, 10 CFEM proteins that have been identified in Aspergillus fumigatus [16], Botryotinia fuckeliana [13], M. oryzae [17], and Sclerotinia sclerotiorum [13], respectively. An average of 16 CFEM proteins were obtained from 12 released Fusarium oxysporum genomes by comparative analysis [18]. Most recently, nine CFEM effector candidates were identified from wheat leaf rust fungus Puccinia triticina [19]. Function studies indicated that CFEM-containing proteins involved in different functional categories [14-16,20-22]. In addition to their function as cell-surface receptors or signal transducers, or as adhesion molecules in host-pathogen interactions [12], some CFEM-containing proteins are proposed to play important roles in pathogenesis in some phytopathogenic fungi. PTH11 and ACI1 proteins produced by Magnaporthe grisea are required for appressorium development and subsequently plant infection $[23,24]$. Deletion of the CFEM domain of PTH11 led to defects in the differentiation of appressoria and appressoria-like structures in M. oryzae during the initiation of the blast disease in rice [17]. Target deletion of BcCFEM1 in Botrytis cinerea suggested that the gene contributes to conidial production and stress tolerance, and most importantly the gene disrupt mutants resulted in decreased virulence on French bean (Phaseolus vulgaris) leaves, indicated that it was involved in pathogenicity [25]. Recently, 
the study by Arya et al. indicated that Bcin07g03260 (a non-GPCR membrane-bound CFEM protein) deletion mutants of $B$. cinerea also showed significantly reduced progression of a necrotic lesion on tomato (Solanum lycopersicum) leaves [26].

Many studies on identification and characterization of pathogenicity associated genes in F. graminearum have been reported. However, the underlying functions and mechanisms by which CFEM proteins act remain largely unknown in this pathogen. To our best knowledge, FGSG_03599 is the only CFEM gene that has been functionally characterized in the pathogen [27]. The status of systemic identification and function analysis of CFEMcontaining proteins in F. graminearum are serious deficiencies. Considering the various functions of CFEM proteins in fungi, the objectives of the current study were to conduct a comprehensive bioinformatics analysis of CFEM proteins in F. graminearum based on the updated and re-annotated genome resource of $\mathrm{PH}-1$ isolate [28].

\section{Materials and Methods}

\subsection{Culture of Fungal Strain}

The F. graminearum strain PH-1 (chemotype 15ADON, isolated on corn from Lansing, MI, USA) used throughout this study was routinely maintained on Potato Dextrose Agar (PDA) medium at $25^{\circ} \mathrm{C}$ in the dark, unless otherwise specified.

Fresh spores of $\mathrm{PH}-1$ were generated in $\mathrm{CMC}$ (Per liter, the medium contained, $0.5 \mathrm{~g}$ $\mathrm{NH}_{4} \mathrm{NO}_{3}, 0.5 \mathrm{~g} \mathrm{KH}_{2} \mathrm{PO}_{4}, 0.5 \mathrm{~g} \mathrm{MgSO}_{4} \cdot 7 \mathrm{H}_{2} \mathrm{O}, 0.5 \mathrm{~g}$ yeast extract, $7.5 \mathrm{~g}$ carboxymethyl cellulose-Na salt) medium with a $12: 12 \mathrm{~h}$ light/dark cycle at $28^{\circ} \mathrm{C}$ for 5 days with continuous shaking at $200 \mathrm{rpm}$. The conidial suspension was filtered through a two-layer Miracloth (Merck, Darmstadt, Germany) and centrifuged at $5000 \mathrm{rpm}$ for $10 \mathrm{~min}$. The harvested conidia were resuspended in sterile water and adjusted to $10^{5}$ conidia $/ \mathrm{mL}$ with the aid of a hemacytometer. Sterile cotton strips were soaked in the conidial suspension for inoculation as described by Zhang et al. [29].

\subsection{Plant Growth Conditions and Inoculation}

The wheat cultivar Ningmai-13, a moderately resistant variety to FHB widely cultured in Jiangsu and Zhejiang, China, was used for the coleoptile infection assay according to Wu et al. [30] with modifications. Seeds were surface sterilized, washed, and germinated as described by Hao et al. [27]. Three days after seed sowing, the top 2-3 $\mathrm{mm}$ of the coleoptiles were removed, and the wounds were wrapped in cotton strips [29]. Mock inoculation using distilled water was carried out in parallel. After inoculation, the seedlings were grown in a growth chamber at $25^{\circ} \mathrm{C}$ and $90 \%$ humidity. A scheme for the inoculation protocol can be found in Supplementary Figure S1.

\subsection{RNA Extraction and Microarray Hybridization}

F. graminearum-inoculated coleoptiles were collected at 7 days post-inoculation (dpi). For each treatment, ten coleoptiles were collected and combined as one sample. Three independent biological replicates were performed for microarray assay. To prepare samples of mycelium grown in vitro for $7 \mathrm{~d}$, cotton strips soaked the conidial suspension were transferred onto PDA plates with cellophane paper and cultivated at $25^{\circ} \mathrm{C}$ for $7 \mathrm{~d}$. Three replicates were included in control treatments. Harvested samples were immediately transferred into liquid nitrogen. RNA samples were collected from the F. graminearuminoculated coleoptiles and mycelium grown on PDA plates was served as control in microarray analysis.

Total RNA was isolated using Trizol Reagent (Cat\#15596-018, Life technologies, Carlsbad, CA, USA) according to the manufacturer's instructions. The quality of RNA samples was assessed using an RNA 6000 Pico Assay Kit with a 2100 BioAnalyzer (Agilent Technologies, Santa Clara, CA, USA). Prior to labeling, qualified total RNA was purified by a RNeasy mini kit (Cat\#74016, QIAGEN, Hilden, Germany) and a RNase-Free DNase Set (Cat\#79254, QIAGEN, Hilden, Germany), according to the manufacturer's guidelines. Chip hybridization, washes, and scanning were performed as described by Zhang et al. [31]. 


\subsection{Bioinformatic Analysis of Common in Fungal Extracellular Membrane (CFEM)-Containing} Proteins in F. Graminearum

\subsubsection{Identification of CFEM-Containing Proteins in F. Graminearum Genome}

To identify the CFEM-containing proteins in the F. graminearum genome (hereafter mentioned as FgCFEM), the previously reported CFEM-containing protein FGSG_03599 was used as query in EnsemblFungi database (http://fungi.ensembl.org/index.html, accessed on 20 September 2021). All obtained proteins were further examined for the presence of the CFEM domain using the PFAM tool in the SMART website (http:/ / smart. embl-heidelberg.de/, accessed on 20 September 2021). The set of identified FgCFEM domains was extracted and used to BLAST the F. graminearum proteins again to find all related sequences.

To examine the distribution of FgCFEM gene features on F. graminearum chromosomes, we mapped the 23 FgCFEM genes on chromosomes. Chromosome location images were generated using Mapchart software V2.32 [32] to localize putative CFEM proteins of $F$. graminearum.

\subsubsection{Signal Peptide, Transmembrane Domain, and Subcellular Localization Prediction}

The SignalP 5.0 Server and TargetP 2.0 Server (https:/ / services.healthtech.dtu.dk/, accessed on 20 September 2021) were used to analyze and predict N-terminal signal peptides (SP) and subcellular localization for predicting protein amino acid sequences, respectively. Additionally, transmembrane regions of CFEM proteins were predicted based on TMHMM (http://www.cbs.dtu.dk/services/TMHMM/, accessed on 20 September 2021). Subcellular localization prediction of CFEM-containing proteins was performed with both TargetP 2.0 Server (https://services.healthtech.dtu.dk/service.php? TargetP-2.0, accessed on 20 September 2021) and Wolf Psort (https://wolfpsort.hgc.jp/, accessed on 20 September 2021).

\subsubsection{Phylogenetic Analysis and Multiple Sequences Alignment}

The AA sequences of CFEM, CFEM_DR domains, and the matured proteins (without SP) were used to create multiple protein sequence alignments using ClustalW using default settings. The neighbor-joining method was used to construct the phylogenetic tree based on AA sequence of domains using MEGA 5.0 [33]. The reliability of the nodes of the tree was evaluated by non-parametric bootstrapping using 1000 pseudo-replicates.

\subsubsection{Domain Analysis of FgCFEM Proteins}

The CFEM domain is a unique domain in fungi and may affect fungal infection and developmental processes. Recently, a new conserved motif, CFEM_DR, was identified by Ling et al. [18] in some CFEM-containing proteins of F. oxysporum. The expression results suggested that some CFEM_DR proteins might be associated with pathogenicity. Therefore, in this study we conducted the motif analysis, including but not limited to CFEM_DR motif, for all the identified CFEM proteins in F. graminearum. For domain detection, the MEME 4.0 software (https:/ / meme-suite.org/meme/tools/meme, accessed on 20 September 2021) was used [34].

\subsubsection{Glycosylphosphatidylinositol (GPI) Modification Site Prediction}

The potential GPI modification sites were predicted using the "GPI Modification Site Prediction in Fungi" tool on the website (https://mendel.imp.ac.at/gpi/fungi_server.html, accessed on 20 September 2021) [35].

\subsubsection{Analysis of Candidate Effectors of CFEM-Containing Proteins}

Based on previous studies by Brown et al. [36], Lu and Edwards [10], proteins containing signal peptides and protein sequences no more than $300 \mathrm{AA}$ without predicted transmembrane regions were considered as potential effectors in this study. The effector probability for each of the secreted protein was further evaluated using EffectorP [37]. 


\section{Results}

\subsection{Bioinformatics Identification of CFEM Proteins in F. Graminearum}

We searched the F. graminearum proteome for CFEM-containing proteins on the basis of their similarity to known CFEM protein. The proteins retrieved in this search were used to BLAST the F. graminearum proteins again to find all related sequences. A total of 22 CFEM proteins (FgCFEM1-22) were found in the F. graminearum genome (Table 1). Based on SMART analysis, the presence of the CFEM domain in these proteins was further verified.

Previously, Zhang et al. [29] analyzed the expression of 21 CFEM genes in F. graminearum during the infection process in wheat. Of the genes analyzed in Zhang et al. [29] the homology of one CFEM gene, FGSG_02840, was not found in our BLAST analysis. According to SMART analysis, we confirmed that the protein encoded by FGSG_02840 contains a CFEM domain, so the homology of this gene (new accession number FGRAMPH1_01G11435) was included in our study and named as FgCFEM23. Thus, it was predicted that there are 23 CFEM proteins encoded in the genome of F. graminearum genome (Table 1 and Figure 1). The different numbers of predicted CFEM proteins between the present research and previous studies may result from the newer genome database version. Among the 23 proteins, all were annotated as "hypothetical proteins" in the Ensembl Fungus Database. Only one CFEM domain was found in each FgCFEM proteins (Figure 1).

(a)

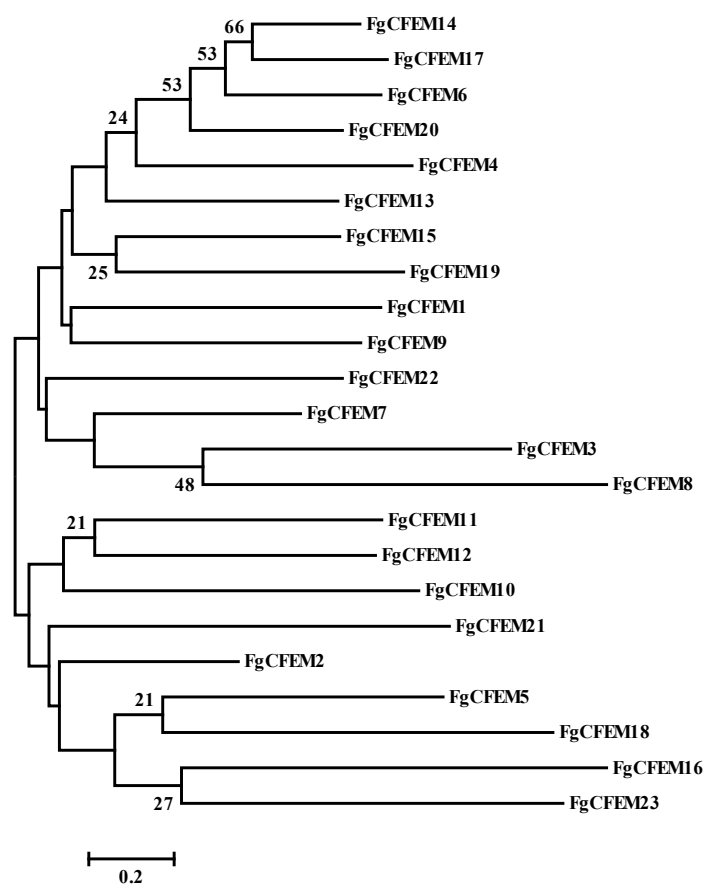

(b)

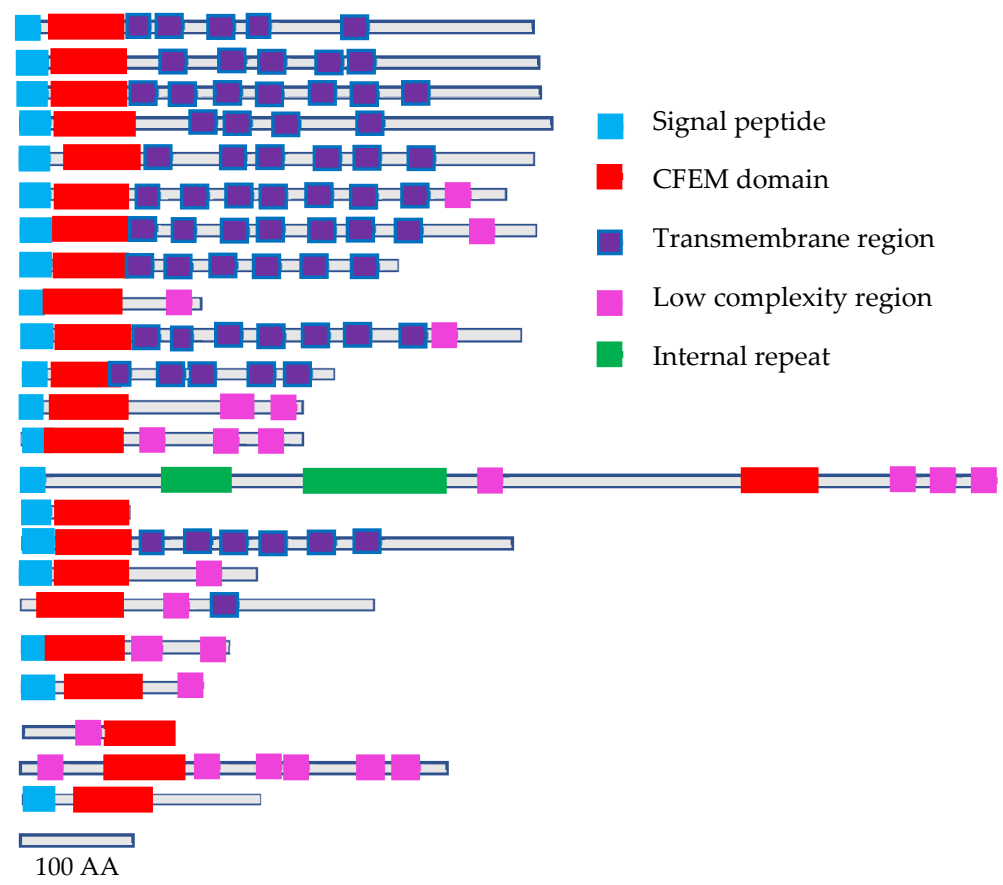

Figure 1. Phylogenetic analysis (a) and structure diagram (b) of the CFEM (Common in Fungal Extracellular Membrane) proteins in F. graminearum. A phylogenetic tree of adjacent connections was constructed based on amino acid sequences of FgCFEMs. The numbers on the nodes represent the percentage of their occurrences in the 1000 bootstrap replicates; the results show that more than $20 \%$ of the nodes are supported. The scale bar shows the number of amino acid differences at each site. The gray lines represent the length of each FgCFEM protein, the sky-blue box structure represents the signal peptide localization, red box represents CFEM structural domain, dark blue box represents transmembrane existence, purple represents low-degree complex regional protein, respectively. The scale represents the length of $100 \mathrm{AA}$ in the architecture. 
Table 1. The identification of Common in Fungal Extracellular Membrane (CFEM) proteins repertoire in F. graminearum.

\begin{tabular}{|c|c|c|c|c|c|c|c|c|c|c|c|c|c|}
\hline Name & Protein ID & $\begin{array}{c}\text { Amino Acid } \\
\text { (AA) }\end{array}$ & No. of Cys & $\begin{array}{l}\text { Cys\% in } \\
\text { Matured } \\
\text { Protein }\end{array}$ & $\begin{array}{c}\text { Position of } \\
\text { CFEM Domain }\end{array}$ & SP Cleavage $^{1}$ & $\mathrm{mTP}^{2}$ & SP & Other $^{3}$ & Loc & $\mathrm{TM}^{4}$ no. & GPI-Anchored & Effector \\
\hline FgCFEM1 & FGRAMPH1_ & 501499160 & 10 & $6.99 \%$ & $18-85$ & $17-18$ & 0 & 0.9998 & 0.0002 & $\mathrm{~S}^{5}$ & 0 & $\mathrm{~N}^{139} / \mathrm{G}^{131}$ & $Y^{7}$ \\
\hline FgCFEM2 & FGRAMPH1_ & 305009184 & 8 & $4.79 \%$ & $19-86$ & $17-18$ & 0 & 0.9999 & 0.0001 & $\mathrm{~S}$ & 0 & $\mathrm{~N}^{163} / \mathrm{G}^{164}$ & - \\
\hline FgCFEM3 & FGRAMPH1_- & 305085250 & 9 & $3.90 \%$ & $17-84$ & $19-20$ & 0 & 0.9998 & 0.0002 & $\mathrm{~s}$ & 0 & $\mathrm{~S}^{220} / \mathrm{A}^{228}$ & - \\
\hline FgCFEM4 & FGRAMPH1_ & 305193457 & 12 & $2.73 \%$ & $25-90$ & $17-18$ & 0 & 0.9992 & 0.0008 & -6 & 6 & $\begin{array}{c}3 \mathrm{~A} \\
-\end{array}$ & - \\
\hline FgCFEM5 & FGRAMPH1_ & 305255161 & 8 & $5.80 \%$ & $35-101$ & $20-21$ & 0.0005 & 0.9993 & 0.0002 & $\mathrm{~s}$ & 0 & $\mathrm{~N}^{138} / \mathrm{A}^{139}$ & $\mathrm{Y}$ \\
\hline FgCFEM6 & FGRAMPH1_ & G05701 463 & 18 & $4.05 \%$ & $28-92$ & $19-20$ & 0.0001 & 0.9925 & 0.0075 & - & 7 & & - \\
\hline FgCFEM7 & FGRAMPH1_ & G10249 207 & 8 & $4.17 \%$ & $17-84$ & $15-16$ & 0 & 0.9999 & 0.0001 & $\mathrm{~s}$ & 0 & $\mathrm{G}^{176} / \mathrm{G}^{186}$ & - \\
\hline FgCFEM8 & FGRAMPH1_ & 310975864 & 22 & $2.60 \%$ & $640-705$ & $17-18$ & 0 & 0.9996 & 0.0003 & $\mathrm{~s}$ & 0 & & - \\
\hline FgCFEM9 & FGRAMPH1_ & G13033 440 & 15 & $3.58 \%$ & $23-87$ & $21-22$ & 0.0003 & 0.7624 & 0.2374 & - & 8 & & - \\
\hline FgCFEM11 & FGRAMPH1_ & G13253 95 & 10 & $12.99 \%$ & $29-92$ & $18-19$ & 0 & 1 & 0 & $\mathrm{~s}$ & 0 & - & $\mathrm{Y}$ \\
\hline FgCFEM12 & FGRAMPH1_ & G13513 435 & 17 & $4.10 \%$ & $26-90$ & $20-21$ & 0 & 0.9991 & 0.0008 & - & 6 & - & - \\
\hline FgCFEM13 & FGRAMPH1_ & G13985 430 & 18 & $4.38 \%$ & $25-88$ & $19-20$ & 0 & 0.9993 & 0.0007 & - & 7 & - & - \\
\hline FgCFEM14 & FGRAMPH1 & G15521 458 & 15 & $3.42 \%$ & $26-90$ & $19-20$ & 0.0004 & 0.9986 & 0.001 & - & 5 & - & - \\
\hline FgCFEM15 & FGRAMPH1 & G16401458 & 9 & $2.05 \%$ & $26-90$ & $20-21$ & 0.0005 & 0.9338 & 0.0657 & - & 7 & 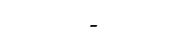 & - \\
\hline FgCFEM16 & FGRAMPH1_ & G17281 379 & 8 & $2.11 \%$ & $73-142$ & -6 & 0.2608 & 0.0319 & 0.7073 & $\mathrm{~S}$ & 0 & $\mathrm{~N}^{355} / \mathrm{S}^{347}$ & - \\
\hline FgCFEM17 & FGRAMPH1_ & G18835 461 & 15 & $3.40 \%$ & $28-92$ & $20-21$ & 0.0009 & 0.9978 & 0.0013 & - & 5 & - & - \\
\hline FgCFEM18 & FGRAMPH1_ & G21361 129 & 7 & $5.43 \%$ & $65-125$ & - & 0.0007 & 0.4664 & 0.5329 & $\mathrm{~s}$ & 0 & - & Y \\
\hline FgCFEM19 & FGRAMPH1 & 321947334 & 9 & $2.87 \%$ & $24-88$ & $20-21$ & 0 & 0.9543 & 0.0457 & - & 6 & - & - \\
\hline FgCFEM20 & FGRAMPH1_ & G25789 472 & 18 & $3.97 \%$ & $27-96$ & $19-20$ & 0.0001 & 0.9956 & 0.0043 & - & 4 & - & - \\
\hline FgCFEM21 & FGRAMPH1_ & 326539312 & 8 & $2.56 \%$ & $9-83$ & - & 0 & 0 & 1 & - & 1 & - & - \\
\hline FgCFEM22 & FGRAMPH1 & G08575 280 & 8 & $3.10 \%$ & $26-88$ & $22-23$ & 0 & 0.906 & 0.094 & - & 5 & 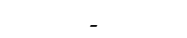 & - \\
\hline FgCFEM 23 & FGRAMPH1_ & G11435 189 & 10 & $5.92 \%$ & $28-95$ & $20-21$ & 0 & 0.9994 & 0.0006 & $\mathrm{~s}$ & 0 & $\mathrm{~N}^{168} / \mathrm{G}^{169}$ & $\mathrm{Y}$ \\
\hline
\end{tabular}

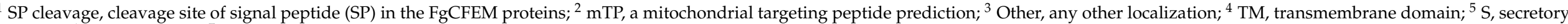
pathway; ${ }^{6}-$, no prediction; ${ }^{7} \mathrm{Y}$, prediction is the effector. 
The full length (including SP) of these proteins ranged from 95 to 864 AA, of which FgCFEM11 is the shortest protein, accounting for 95 AA, and FgCFEM8 is the largest protein, accounting for $864 \mathrm{AA}$. On the other hand, the predicted mature proteins of the 23 CFEMs consisted of 77 to 847 AA with a majority (13 proteins) $<400$ AA. The smallest mature CFEM is FgCFEM11, which consisted of less than 100 AA. Cysteine residues in the mature proteins varied in number from 7 to 22 with a majority (18 proteins) $\leq 15$, while the percentage of cysteine in mature proteins is $2.05 \%$ to $12.99 \%$ with most (18 proteins) $<5 \%$. Phylogenetic and multiple sequence alignment analysis revealed sequences conservation, and the eight cysteine residues in particular are well-conserved in most of these CFEM domains (Figure 2), which may be involved in the formation of disulfide bonds and play significant roles in the structure and function of the protein.

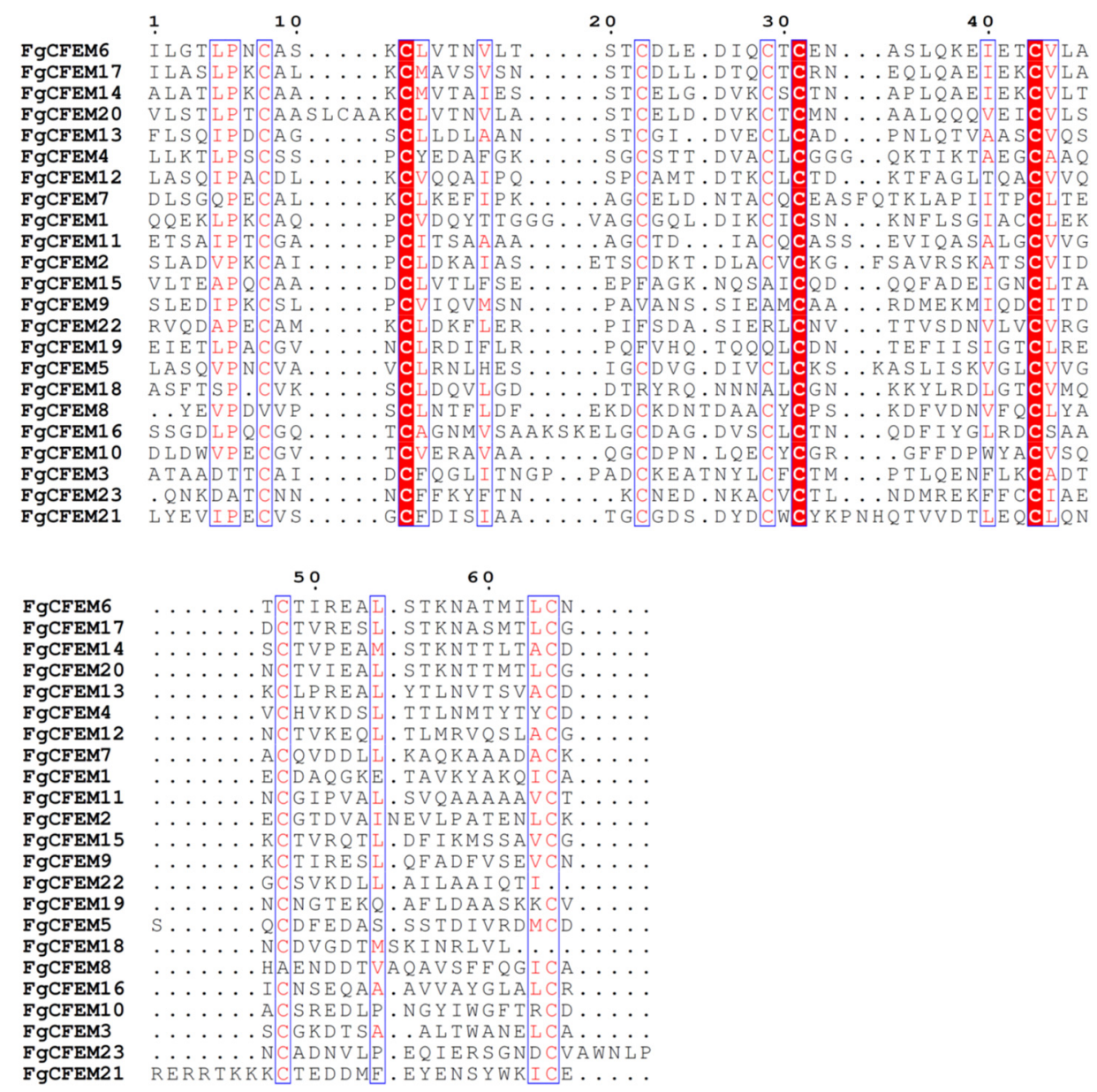

Figure 2. Multi-sequence alignment of the CFEM proteins in F. graminearum. The alignment was performed using ClustalW program, and the conserved amino acids are highlighted in red color. Red color font: conserved AA in some but not all the FgCFEMs, red color background: conserved AA in all the 23 FgCFEMs.

\subsection{Chromosomal Distribution of CFEM-Containing Genes}

To determine the chromosomal distribution of putative CFEM-containing proteins, chromosome map was constructed from F. graminearum (Figure 3). The putative 23 FgCFEM genes are distributed among all four chromosomes, and chromosome 2 encoded the highest number, 9 genes of putative FgCFEM genes, followed by chromosome 1, 3, and 4, encoding 7,5 , and 2 genes, respectively. Overall, the genes encoding the 23 FgCFEMs appeared to be distributed randomly in the genome as they can be found in all four chromosomes with comparable numbers. 


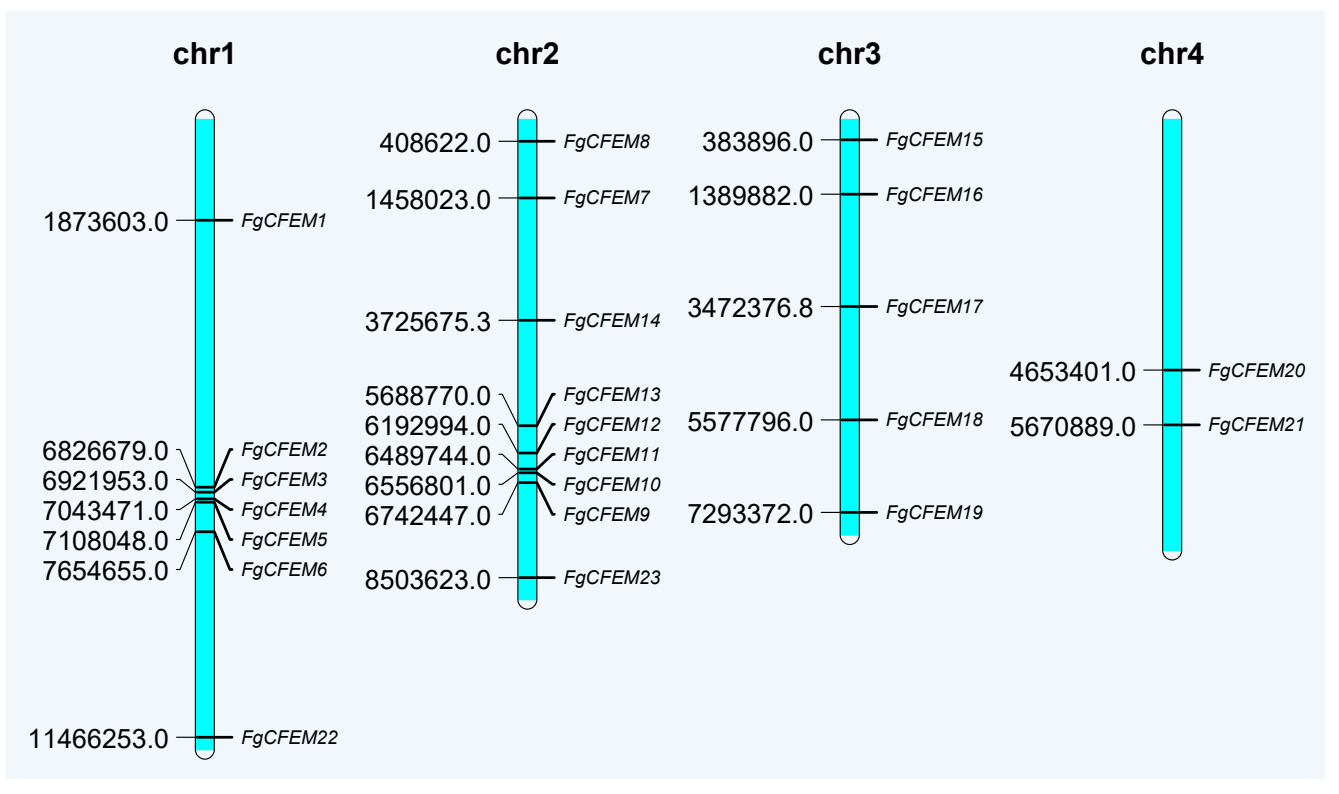

Figure 3. Mapping of the CFEM genes on F. graminearum (strain PH-1) chromosomes.

\subsection{Feature Characterization of CFEM-Containing Proteins}

We used the SignalP 5.0 Server to predict all CFEM protein signal peptides in F. graminearum and preliminarily determine the composition of these proteins. The results showed that 20 of the 23 CFEM proteins contained a signal peptide (Table 1 and Figure 1), whose sequence was a small segment of amino acid at the $\mathrm{N}$-terminal. The SMART analysis result indicated that the initial amino acids between 15 to 23 encoded the signal peptides. No SP sequence was predicted in the remaining 3 FgCFEMs (FgCFEM16, 18, and 21) (Table 1).

TargetP 2.0 and Wolf Psort analysis showed that 11 FgCFEM proteins were predicted as secretory proteins which could be secreted out of the cell through the secretion pathway of F. graminearum (Table 1).

According to TMHMM prediction, it demonstrated that 4-8 transmembrane regions were identified in 11 FgCFEMs and only one transmembrane region was found in FgCFEM21. No transmembrane region was found in the other 11 CFEM proteins (Table 1).

Further analysis of the 20 SP-containing FgCFEMs shows that 11 proteins have transmembrane domains and belonged to transmembrane proteins. The other nine CFEM proteins do not contain transmembrane domains and belong to secretory proteins. Moreover, of the $20 \mathrm{SP}$-containing proteins, nine were annotated by EnsemblFungi that consist of less than $300 \mathrm{AA}$ in full-length (Table 1).

GPI Modification Site Prediction (http:/ /mendel.imp.ac.at/gpi/plant_server.html, accessed on 20 September 2021) was used to predict potential GPI modification site. The result demonstrated that two putative GPI modification sites were found in eight CFEMs, and the two identified amino acids are predicted to be the best and second best of potential GPI-modification sites, respectively (Table 1). For example, the amino acids $\mathrm{N}^{139}$ and $\mathrm{G}^{131}$, $\mathrm{N}^{163}$ and $\mathrm{G}^{164}, \mathrm{~S}^{220}$ and $\mathrm{A}^{228}$ are the best and second best of potential GPI-modification sites in FgCFEM1, FgCFEM2, and FgCFEM3, respectively. No GPI modification site was predicted in the other 15 CFEMs. The results indicated that some F. graminearum CFEMs contain putative GPI-anchored sites, which are possibly anchored to the outer layer of the plasma membrane through an anchor or transferred to the cell wall as other GPI-anchored proteins in fungi [38]. According to previous studies, GPI-anchored proteins on the fungal cell wall have important effects on fungal adhesion, morphological transformation and cell wall synthesis, and microbial adhesion is one of the most important determinants of its pathogenicity. Therefore, to some extent, these CFEM proteins in F. graminearum are more likely to be associated with fungal disease. 


\subsection{Identification of Potential CFEM Effectors in F. Graminearum}

Protein effectors are most often secreted via the conventional endoplasmic reticulumGolgi apparatus rote, so normally they must contain an N-terminal secretion signal. Effector candidates can thus be identified bioinformatically by the presence of this signal [39]. Among the 23 proteins, 20 were found to contain an N-terminal signal peptide, which were preliminarily considered as the sources of secretory proteins. Combined with the subcellular localization and TMHMM analysis results, 11 FgCFEMs (FgCFEM1, 2, 3, 5, 7, 8, $10,11,16,18,23)$ were selected as putative effectors based on the criteria that the predicted mature proteins belong to secretory proteins and do not contain transmembrane regions.

All the 23 CFEMs were also evaluated using EffectorP [37] individually to predict their possibility as effectors. The results indicated that five proteins (FgCFEM1, 5, 11, 18 , and 23) were predicted to be candidate effectors, which are all from the aforementioned 11 FgCFEMs. Our prediction result is consistent with the previous studies that FgCFEM11 (the homology of FGSG_03599), one of the CFEM proteins in F. graminearum, has been identified as an effector and functionally analyzed, which may be involved in plant infection [10,27]. Among the five candidate effectors, interestingly, FgCFEM18 is different from the other four CFEM proteins which contain no SP sequence by SignalP 5.0 prediction analysis. So probably FgCFEM18 is secreted through a novel pathway. Subsequently, the protein sequence of FgCFEM18 was submitted to the SecretomeP 2.0a Server (http:/ / www.cbs.dtu.dk/services/SecretomeP/, accessed on 20 September 2021), and the result indicated that FgCFEM18 is a non-classically secreted protein with a NN-score 0.863 which means that the protein is probably secreted in non-classical pathways.

\subsection{Phylogenetic Analysis of CFEM Proteins from F. Graminearum}

When compared at AA level, most CFEM proteins lacked significant similarities to each other. To elucidate the evolutionary relationships among the 23 CFEMs in F. graminearum, the sequences of CFEM domains were extracted for phylogenetic analysis. Moreover, two well-studied CFEM-containing proteins, PTH11 in M. grisea and BCCFEM1 in B. cinerea, respectively, were included to determine any relationships (Figure 4).

According to the phylogenetic tree, all the 25 CFEMs can be divided into four major clades (Figure 4). Among them, FgCFEM14, 17, and 20 have relatively high homology, and the similarity is more than $52 \%$. Additionally, the homology of FgCFEM11 and BcCFEM1 exceeded $42 \%$ and the two domains were clustered into a sub-group in phylogenetic analysis (Figure 4 ) while low CFEM domain identity $(\leq 25 \%)$ was observed between PTH11 and all the FgCFEMs.

The structure of CFEM domains was also analyzed. The 23 CFEM domain sizes ranged from 61 to 75 AA with a majority (seven domains) at 65 AA. Among these CFEM domains, 17 domains contain the eight conserved cysteines which could form four disulfide bones to stabilize the whole protein structure [40]. However, two conserved cysteines were missed in the CFEM domains of FGCFEM8, 9, 15, and 19, and three cysteines were missed in FgCFEM18 and FgCFEM22 (Figure 2).

\subsection{Conserved Motif Analysis}

To reveal the potential conserved sequences of the CFEMs in F. graminearum, the MEME motif search tool was used to identify candidate motifs of these 23 proteins. By multiple sequence alignments of all $23 \mathrm{CFEM}$ proteins, three blocks of conserved sequences outside the CFEM domain were detected in nine protein (FgCFEM4, 6, 9, 12, 13, 14, 15, 17, 20) (Figure 5). Each block contained a conserved motif (Figure 5). For examples, motifs 1, 2, and 3 were conserved in $x D x P x K x F x G x R, x W x E x R x$, and KxFxIFx patterns respectively. However, in FgCFEM19 and FgCFEM22 only motifs 1 and 3 were detected and no motif 2 was identified. For the other members, we failed to find any conserved motifs outside the CFEM domain, indicating the conserved cysteine amino acid sequence is the only feature for these proteins. Motif search results indicated that PHT11, the well studied CFEM 
protein in $M$. oryzae, contains all the three motifs, while none of these motifs were found in BcCFEM1 in B. cinerea.

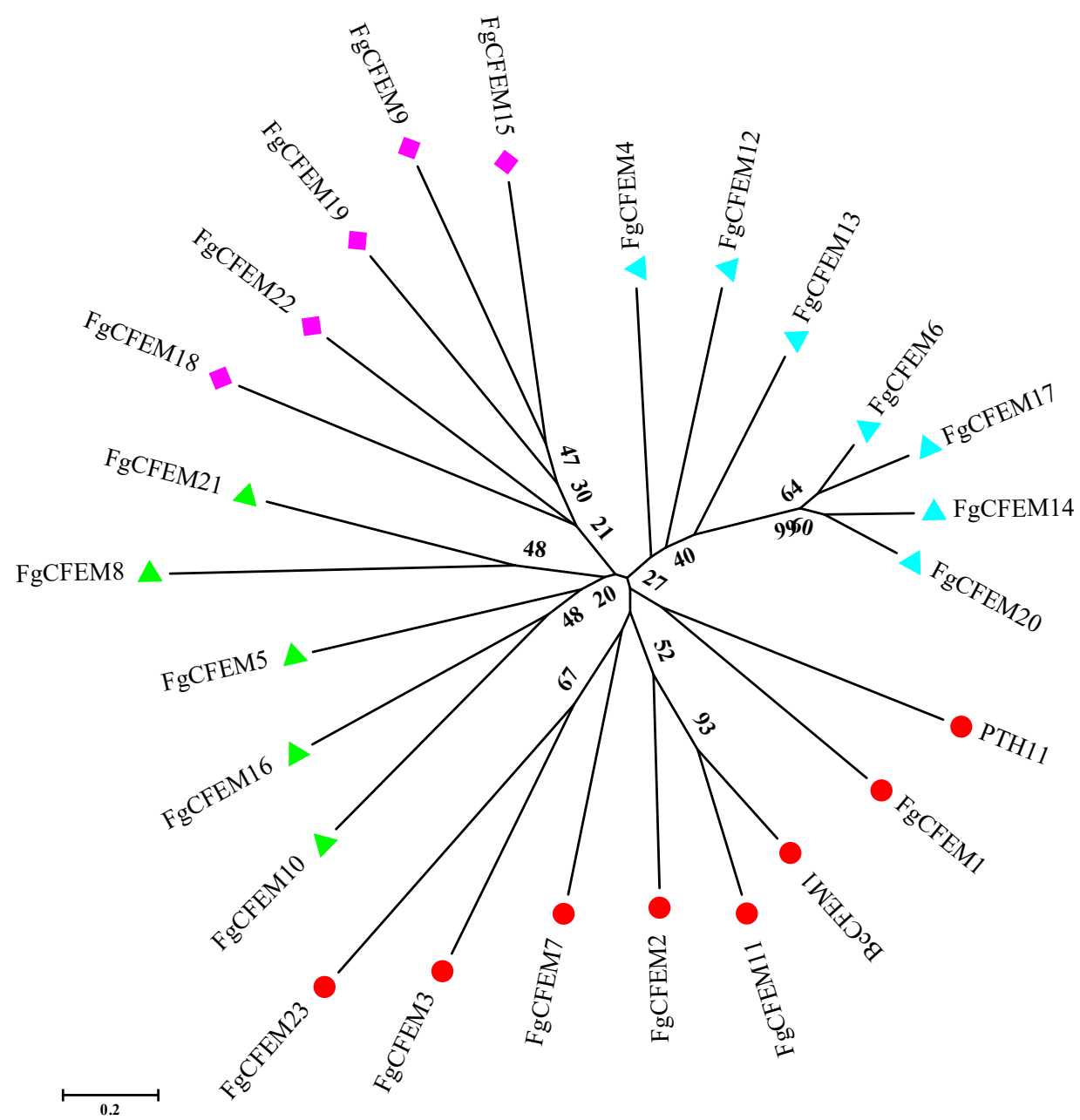

Figure 4. Phylogenetic analysis of the extracted CFEM domains of CFEM proteins in F. graminearum, PTH11 in M. oryzae and BCCFEM1 in B. cinerea. A phylogenetic tree of adjacent connections was constructed based on amino acid sequences of FgCFEMs. The numbers on the nodes represent the percentage of their occurrences in the 1000 bootstrap replicates; the results show that more than $20 \%$ of the nodes are supported. The scale bar shows the number of amino acid differences at each site. The different colors indicate different groups.

As shown in Figure 5, the conserved motif 1 resides on the C-terminal end of FgCFEM proteins and contains about $50 \mathrm{AA}$, six of which are conserved. The conserved residues of motif 1 detected in this study were consistent with previous work in which they were identified from F. oxysporum by Ling et al. [18], indicating that motif 1 belongs to CFEM_DR motif. Motif 2 and motif 3 are the novel conserved motifs. The conserved motif 3 resides in the middle of CFEM proteins and contains about $18 \mathrm{AA}$, four of which are conserved (KxFxIFx). The conserved motif 2 resides on the C-terminal end of CFEM proteins and contains about 29 AA, while only three conserved residues (xWxExRx) are detected. We referred to motif 2 and motif 3 as the WR motif and KF motif, respectively, in this study according to the first and last conserved amino acids in the motifs (WR and KF, respectively); the CFEM proteins containing the motif were referred as CFEM_WR and CFEM_KF proteins. Multiple sequence alignment of WR and KF motifs were given in Supplementary Figure S2. The identification of the novel motifs in some of the CFEM proteins indicated they might have divergent functions from the other CFEM proteins. 


\subsection{The Transcriptometrics Analysis of CFEM Genes in Planta}

RNA derived from these samples was hybridized to the F. graminearum Affymetrix GeneChip. Transcriptional profiling of the CFEMs was carried out using a custom designed Agilent oligomer array, and the array contained up to three individual 60-mers for each gene. A total of 13,382 oligomers representing 13,382 fungal transcripts were perceived in the experiments. Microarray analyses revealed that the expression patterns of the 23 CFEMs differed greatly. The expression heatmap of these 23 FgCFEM genes are as shown in Figure 6. Specifically, the results of our microarray analysis showed that all the 23 CFEM genes were expressed at $7 \mathrm{dpi}$, with 7 genes $(F g C F E M 6,8,11,12,13,17,23)$ significantly up-regulated and six genes ( $F G C F E M 2,7,14,15,16,19)$ significantly downregulated in planta (fold change, $F C \geq 2$ ). While no significant difference was found for the other 10 genes compared with in planta and in vitro.

(a)

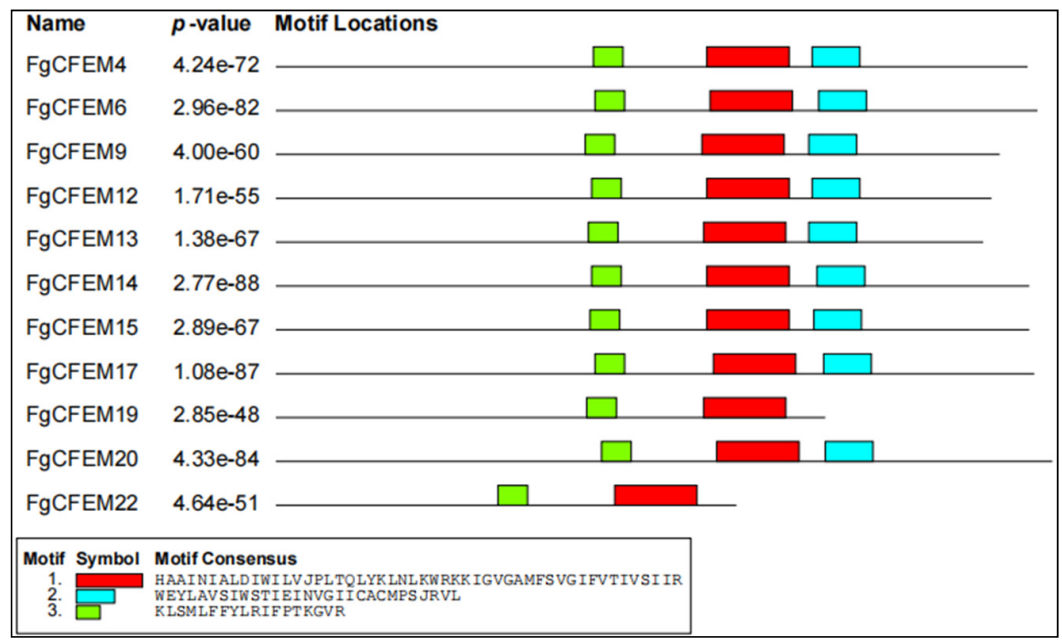

(b)

Motif 1

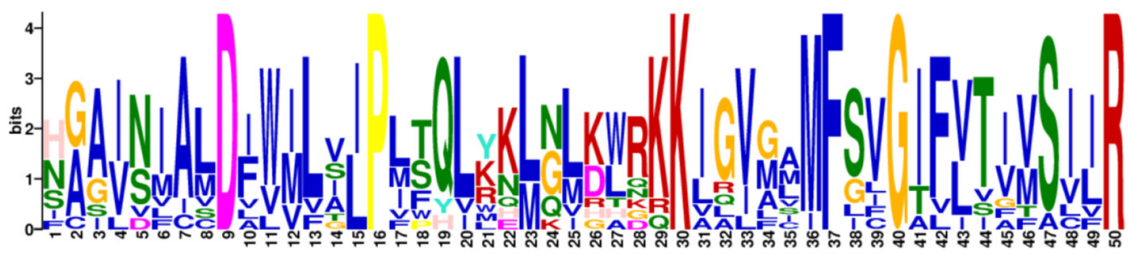

Motif 2

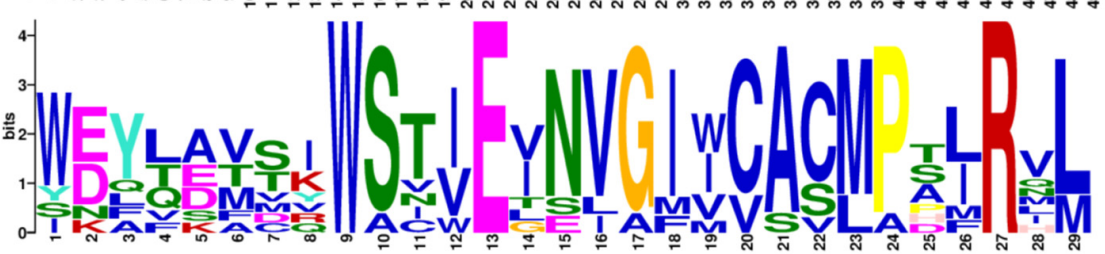

Motif 3

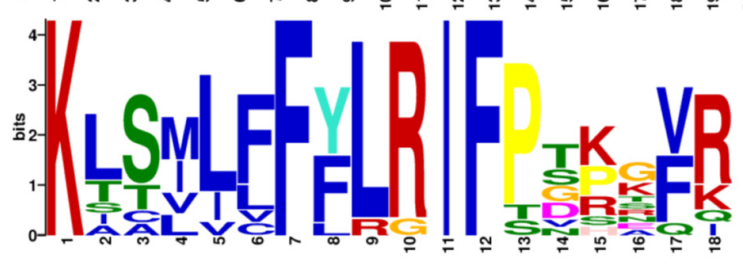

Figure 5. Identification of conserved motifs outside of CFEM domains by the MEME motif search tool. (a) Locations of new motifs identified. The black line represents the length of CFEM proteins, the red rectangle represents the motif 1 , the blue rectangle represents motif 2 , and the green protein represents motif 3 ; (b) a schematic diagram of motif 1 , motif 2 and motif 3 and the conserved AA generated by MEME V4.0 software. 


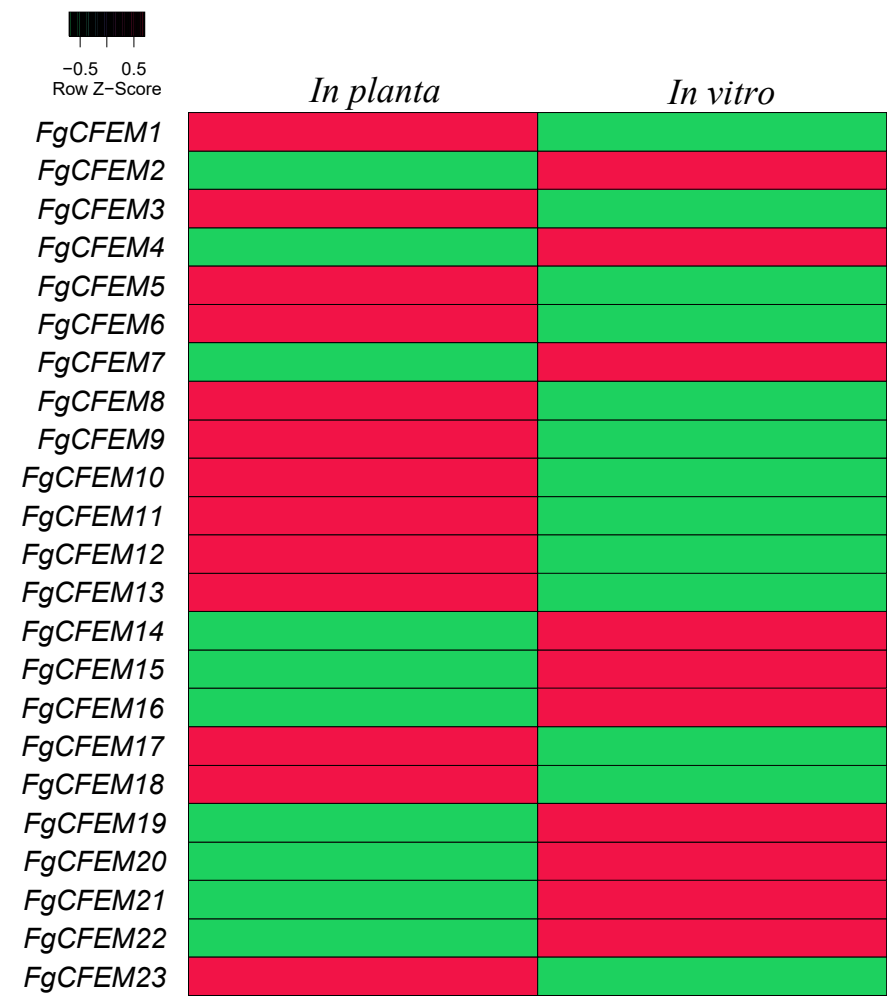

Figure 6. The heat map of FgCFEM genes. Green represents lower expression, red represents higher expression.

On the other hand, a genome-wide analysis of SSCPs was conducted by Lu and Edwards [10], and six CFEM-containing proteins (FgCFEM1, 2, 5, 7, 10, 11) were detected. Among the six genes, FgCFEM1, 2, and 7 were constitutively expressed but no significant differences were observed compared with in planta and in vitro, whereas none of transcripts of FgCFEM5 and FgCFEM10 were detectable in planta infection or in vitro. Only the expression of FgCFEM11 (the homology of FGSG_03599) was up regulated in comparison with those in fungal cultures. In our study, the expression pattern of FgCFEM11 is consistent with the previous studies in Lu and Edwards [10].

Of the seven up-regulated FgCFEM genes identified in microarray analysis, only FgCFEM11 and FgCFEM 23 were predicted to be effector candidates by bioinformatics analysis. The expression levels of the two genes in planta are 367.49 and 8.26 times higher, respectively, than in vitro. Both FgCFEM11 and FgCFEM23 contain a SP sequence at the $\mathrm{N}$-terminal and no transmembrane region was identified for the two proteins. Considering the protein sizes, FgCFEM11 and FgCFEM23 accounting for 95 and 189 AA, respectively, indicating they are typical SSCPs. All the eight spaced cysteines were conserved in the two CFEM proteins by sequence alignment analysis. Combined with the transmembrane region prediction, subcellular localization analysis, EffectorP prediction and microarray results, we extrapolated that FgCFEM11 and FgCFEM 23 are the two effectors among the CFEM-containing proteins.

\section{Discussion}

CFEM domain is unique to fungi and CFEM-containing proteins were found to be enriched in pathogenic fungi [13]. The roles of several CFEM proteins in different fungi were characterized. However, the specific roles of CFEM proteins in F. graminearum remain largely unknown. In the present study, we searched the proteome of $F$. graminearum for CFEM-containing proteins and identified a total of 23 sequences. This is the second largest number of CFEM candidates identified for any fungal species, and the largest number of this class of protein was identified in Colletotrichum graminicola [41]. However, only 
FGSG_03599, which is associated with virulence, has been reported [27]. All the identified CFEM-containing proteins in F. graminearum were annotated as hypothetical proteins in the new version of the strain PH-1 genome database. Sequence alignments revealed that most of the CFEM domains contain eight shared cysteine residues. The extreme aminoterminal and the carboxy-terminal sequences flanking this domain were divergent. This is consistent with other observations that sequences conservation is typically limited to the CFEM domains in CFEM-containing proteins.

CFEM-containing proteins involved in different functional categories. CFEM-containing proteins have been found exclusively in fungi, especially in Ascomycota and Basidiomycota. For example, CFEM domain was found to participate in various functions mediating different physiological (cell wall stability [16,42]) and infection processes [25,43]. Studies of phytopathogenic fungi demonstrated the role of CFEM-containing proteins involved in different aspects of virulence. For example, the PTH11 and PTH11-like proteins are required for proper development of the appressoria, and pathogenicity in M. oryzae [17,43]. Similarly, in M. grisea [23], the adenylate cyclase (MAC1) CFEM-containing protein was shown to regulate appressorium formation. However, the underlying functions and mechanisms by which CFEM proteins act remain largely unknown in FGSC. Therefore, further research is both necessary and needed to answer these questions.

Among the 23 CFEM-containing proteins in F. graminearum, 20 were predicted to contain a SP and no SP sequence was detected in the other 3 proteins (FgCFEM16, 18, 21). These three proteins were further subjected to SecretomeP predictions for non-classically secrete proteins, and two of them (FgCFEM16, 21) obtain an NN-score exceeding the threshold (mammalian, 0.6 ) predicted by SecretomeP, indicating that these may be secreted in non-classical pathways. Non-classical secretory proteins were previously reported in secretome analysis of F. graminearum. Among the 69 unique fungal proteins identified, 11 were predicted to be secreted in a non-classical manner in F. graminearum [44]. Similarly, proteins secreted in a non-classical way in other fungi, e.g., Aspergillus fumigates, Candida albicans, Claviceps purpurea, and Saccharomyces cerevisiae have been reported previously $[44,45]$.

The potential modification of CFEM-containing proteins was also analyzed in this study. The result demonstrated that putative GPI modification sites were found in eight CFEMs, indicating these may be GPI-anchored CFEM proteins. GPI-anchored proteins that can anchor to the outer layer of the plasma membrane through a C-terminal GPI anchor are essential for growth, signaling transmission, surface adhesion, and disease pathogenesis in eukaryotic cells [46]. For example, BcCFEM1 encodes a CFEM protein with a putative GPI modification site in pathogen fungus $B$. cinerea. Disruption of this gene results in decreased virulence and increased sensitivity to osmotic and cell wall stress, indicating that BcCFEM1 is required for virulence and plays a key role in stress resistance [25]. The recent work by Arya et al. [26] illustrates a potential new role for a non-GPCR membrane CFEM in pathogenic fungi to control virulence in the fungus $B$. cinerea.

Effectors play critical roles during pathogen and plant interactions. Bioinformatics analysis predicted that there are about 600 effectors in the genome of F. graminearum [36]. Feature analysis indicated that many of these effectors are SCPPs that contain N-terminus signal peptides and lack transmembrane domains. In the study by Lu and Edwards [10], at least 34 SCPPs have been shown to be expressed in infected wheat heads. In this study, microarray analysis result indicated that all the CFEM-containing proteins were expressed during wheat infection, and seven genes were significantly up-regulated with FgCFEM11 has the highest fold change in planta compared with in vitro. Based on the structure features and microarray analysis results, we conjectured that FgCFEM11 and FgCFEM23 are more probably to function as effectors during plant infection and may be involved in pathogenesis.

Conserved motif analysis indicated that 11 of the 23 FgCFEM proteins contained the CFEM_DR motif which recently identified in F. oxysporum [18]. In addition, by constructing phylogenetic tree and using MEME software, two new conserved motifs were identified by us in these 11 FgCFEM proteins. Future studies on the functional analysis of the 
two motifs should be conducted to investigate their potential roles during pathogen and plant interactions.

\section{Conclusions}

In this study, we identified a total of 23 FgCFEM proteins in F. graminearum genome based on the recently updated and re-annotated genome resource of PH-1 isolate [28]. A comprehensive bioinformatics analysis on signal peptide, transmembrane domain, protein subcellular localization, and potential GPI modification sites of these CFEM proteins was conducted. The results showed that only 10 CFEM proteins were secreted proteins and GPI modification sites were identified in eight proteins. A subset of five effector candidates encoded by CFEMs was predicted using EffectorP [37]. Transcriptome analysis of all the 23 CFEM proteins was performed during infection process and seven CFEM-containing genes were highly expressed in wheat compared with control. Combined with the features characterization of CFEM proteins, we proposed that FgCFEM11 and FgCFEM23 are the two effectors among the CFEM-containing proteins in F. graminearum. Our findings provide a theoretical basis for in-depth analysis for the function of CFEM effectors in phytopathogenic fungi.

Supplementary Materials: The following are available online at https: / www.mdpi.com/article/ 10.3390/jof7100871/s1, Figure S1: A scheme for wheat inoculation protocol, Figure S2: Multiple sequence alignment of WR and KF motifs.

Author Contributions: Methodology, L.C., H.W., J.Y., X.Y., M.Z., Z.Z., Y.F., C.W. and J.W.; software, L.C. and J.W.; validation, L.C., H.W., M.Z. and J.W.; investigation, L.C., H.W. and J.W.; writingoriginal draft preparation, L.C.; writing-review and editing, J.W.; visualization, L.C., H.W. and J.W.; supervision, J.W.; project administration, J.W. and J.Y. All authors have read and agreed to the published version of the manuscript.

Funding: This research was funded by the National Natural Science Foundation of China (grant numbers 31871896, 31901804, and 31401598), Science and Technology Commission of Shanghai Municipality (grant number 21DZ1201300).

Institutional Review Board Statement: Not applicable.

Informed Consent Statement: Not applicable.

Data Availability Statement: The data presented in this study are available on request from the corresponding author. The data are not publicly available because other data from these wholegenome transcriptomes are being used for other analyses to be published independently of this one.

Conflicts of Interest: The authors declare no conflict of interest.

\section{References}

1. Garmendiaa, G.; Pattarinoa, L.; Negrína, C.; Martínez-Silveiraa, A.; Pereyrab, S.; Wardc, T.J.; Vero, S. Species composition, toxigenic potential and aggressiveness of Fusarium isolates causing Head Blight of barley in Uruguay. Food Microbiol. 2018, 76, 426-433. [CrossRef]

2. Starkey, D.E.; Ward, T.J.; Aoki, T.; Gale, L.R.; Corby Kistler, H.; Geiser, D.M.; Suga, H.; Toth, B.; Varga, J.; O’Donnell, K. Global molecular surveillance reveals novel Fusarium head blight species and trichothecene toxin diversity. Fungal Genet. Biol. 2007, 44, 1191-1204. [CrossRef]

3. Trail, F. For blighted waves of grain: Fusarium graminearum in the postgenomics era. Plant Physiol. 2009, 149, 103-110. [CrossRef] [PubMed]

4. Dean, R.; Van Kan, J.A.L.; Pretorius, Z.A.; Hammond-Kosack, K.E.; Pietro, A.D.; Spana, P.D.; Rudd, J.J.; Dickman, M.; Kahmann, R.; Ellis, J.; et al. The Top 10 fungal pathogens in molecular plant pathology. Mol. Plant Pathol. 2012, 13, 414-430. [CrossRef]

5. Desjardins, A.E.; Proctor, R.H.; Bai, G.; McCormick, S.P.; Shaner, G.; Buechely, G.; Hohn, T.M. Reduced virulence of trichothecenenonproducing mutants of Gibberella zeae in wheat field tests. Mol. Plant Microbe Interact. 1996, 9, 775-781. [CrossRef]

6. Jansen, C.; von Wettstein, D.; Schafer, W.; Kogel, K.H.; Felk, A.; Maier, F.J. Infection patterns in barley and wheat spikes inoculated with wild-type and trichodiene synthase gene disrupted Fusarium graminearum. Pro. Natl. Acad. Sci. USA 2005, 102, 16892-16897. [CrossRef] [PubMed]

7. Proctor, R.H.; Hohn, T.M.; McCormick, S.P. Reduced virulence of Gibberella zeae caused by disruption of a trichothecene toxin biosynthetic gene. Mol. Plant Microbe Interact. 1995, 8, 593-601. [CrossRef] [PubMed] 
8. Wang, H.W.; Sun, S.; Ge, W.Y.; Zhao, L.F.; Hou, B.Q.; Wang, K.; Lyu, Z.F.; Chen, L.Y.; Xu, S.S.; Guo, J.; et al. Horizontal gene transfer of Fhb7 from fungus underlies Fusarium head blight resistance in wheat. Science 2020, 368, 844. [CrossRef]

9. Narayanan, S.P.; Lung, S.C.; Liao, P.; Lo, C.; Chye, M.L. The overexpression of OsACBP5 protects transgenic rice against necrotrophic, hemibiotrophic and biotrophic pathogens. Sci. Rep. 2020, 10, 14918. [CrossRef]

10. Lu, S.; Edwards, M.C. Genome-wide analysis of small secreted cysteine-rich proteins identifies candidate effector proteins potentially involved in Fusarium graminearum-wheat interactions. Phytopathology 2016, 106, 166-176. [CrossRef]

11. Appella, E.; Weber, I.T.; Blasi, F. Structure and function of epidermal growth factor-like regions in proteins. FEBS Lett. 1988, 231, 1-4. [CrossRef]

12. Kulkarni, R.D.; Kelkar, H.S.; Dean, R.A. An eight-cysteine-containing CFEM domain unique to a group of fungal membrane proteins. Trends Biochem. Sci. 2003, 28, 118-121. [CrossRef]

13. Zhang, Z.N.; Wu, Q.Y.; Zhang, G.Z.; Zhu, Y.Y.; Murphy, R.W.; Liu, Z.; Zou, C.G. Systematic analyses reveal uniqueness and origin of the CFEM domain in fungi. Sci. Rep. 2015, 5, 13032. [CrossRef]

14. Pérez, A.; Pedrós, B.; Murgui, A.; Casanova, M.; López-Ribot, J.L.; Martínez, J.P. Biofilm formation by Candida albicans mutants for genes coding fungal proteins exhibiting the eight-cysteine-containing CFEM domain. FEMS Yeast Res. 2006, 6, 1074-1084. [CrossRef]

15. Pérez, A.; Ramage, G.; Blanes, R.; Murgui, A.; Casanova, M.; Martínez, J.P. Some biological features of Candida albicans mutants for genes coding fungal proteins containing the CFEM domain. FEMS Yeast Res. 2011, 11, 273-284. [CrossRef]

16. Vaknin, Y.; Shadkchan, Y.; Levdansky, E.; Morozow, M.; Romano, J.; Osherov, N. The three Aspergillus fumigatus CFEM-domain GPI-anchored proteins (CfmA-C) affect cell-well stability but do not play a role in fungal virulence. Fungal Genet. Biol. 2014, 63, 55-64. [CrossRef]

17. Kou, Y.; Tan, Y.H.; Ramanujam, R.; Naqvi, N. Structure-function analyses of the Pth11 receptor reveal an important role for CFEM motif and redox regulation in rice blast. New Phytol. 2017, 214, 330-342. [CrossRef]

18. Ling, J.; Zeng, F.; Gao, Y.X.; Zhang, J.X.; Chen, G.H.; Mao, Z.C.; Yang, Y.H.; Xie, B.Y. Identification of a class of CFEM proteins containing a new conserved motif in Fusarium oxyporum. Physiol. Mol. Plant Pathol. 2015, 89, 41-48. [CrossRef]

19. Zhao, S.; Shang, X.; Bi, W.; Yu, X.; Liu, D.; Kang, Z.; Wang, X.; Wang, X. Genome-wide identification of effector candidates with conserved motifs from the wheat leaf rust fungus Puccinia triticina. Front. Microbiol. 2020, 11, 1188. [CrossRef]

20. Ding, C.; Vidanes, G.M.; Maguire, S.L.; Guida, A.; Synnott, J.M.; Andes, D.R.; Butler, G. Conserved and divergent roles of Bcr1 and CFEM proteins in Candida parapsilosis and Candida albicans. PLoS ONE 2011, 6, e28151. [CrossRef]

21. Weissman, Z.; Kornitzer, D. A family of Candida cell surface haem-binding proteins involved in haemin and haemoglobin-iron utilization. Mol. Microbiol. 2004, 53, 1209-1220. [CrossRef]

22. Weissman, Z.; Shemer, R.; Conibear, E.; Kornitzer, D. An endocytic mechanism for haemoglobin-iron acquisition in Candida albicans. Mol. Microbiol. 2008, 69, 201-217. [CrossRef]

23. Choi, W.; Dean, R.A. The adenylate cyclase gene MAC1 of Magnaporthe grisea controls appressorium formation and other aspects of growth and development. Plant Cell 1997, 9, 1973-1983.

24. DeZwaan, T.M.; Carroll, A.M.; Valent, B.; Sweigard, J.A. Magnaporthe grisea Pth11p Is a Novel Plasma Membrane Protein That Mediates Appressorium Differentiation in Response to Inductive Substrate Cues. Plant Cell 1999, 11, 2013-2030. [CrossRef]

25. Zhu, W.J.; Wei, W.; Wu, Y.Y.; Zhou, Y.; Peng, F.; Zhang, S.P.; Chen, P.; Xu, X.W. BcCFEM1, a CFEM Domain-Containing Protein with Putative GPI-Anchored Site, Is Involved in Pathogenicity, Conidial Production, and Stress Tolerance in Botrytis cinerea. Front. Microbiol. 2017, 8, 1807. [CrossRef]

26. Arya, G.C.; Srivastava, D.A.; Pandaranayaka, E.P.J.; Manasherova, E.; Prusky, D.B.; Elad, Y.; Frenkel, O.; Dvir, H.; Harel, A. Characterization of the role of a non-GPCR membrane-bound CFEM protein in the pathogenicity and germination of Botrytis cinerea. Microorganisms 2020, 8, 1043. [CrossRef]

27. Hao, G.; McMormick, S.; Usgaard, T.; Tiley, H.; Vaughan, M.M. Characterization of three Fusarium graminearum effectors and their roles during fusarium head blight. Front. Plant Sci. 2020, 11, 579553. [CrossRef]

28. King, R.; Urban, M.; Hammond-Kosack, M.C.U.; Hassani-Pak, K.; Hammond-Kosack, K.E. The completed genome sequence of the pathogenic ascomycete fungus Fusarium graminearum. BMC Genom. 2015, 16, 544. [CrossRef]

29. Zhang, X.; Jia, L.; Zhang, Y.; Jiang, G.; Li, X.; Zhang, D.; Tang, W. In planta stage-specific fungal gene profiling elucidates the molecular strategies of Fusarium graminearum growing inside wheat coleoptiles. Plant Cell 2012, 24, 5159-5176. [CrossRef]

30. Wu, A.B.; Li, H.P.; Zhao, C.S.; Liao, Y.C. Comparative pathogenicity of Fusarium graminearum isolates from China revealed by wheat coleoptile and floret inoculations. Mycopathologia 2005, 160, 75-83. [CrossRef]

31. Zhang, Y.; He, J.; Jia, L.; Yuan, T.; Zhang, D.; Guo, Y.; Wang, Y.; Tang, W. Cellular tracking and gene profiling of Fusarium graminearum during maize stalk rot disease development elucidates its strategies in confronting phosphorus limitation in the host apoplast. PLoS Pathog. 2016, 12, e1005485. [CrossRef]

32. Voorrips, R.E. MapChart: Software for the graphical presentation of linkage maps and QTLs. J. Hered. 2002, 93, 77-78. [CrossRef]

33. Tamura, K.; Peterson, D.; Peterson, N.; Stecher, G.; Nei, M.; Kumar, S. MEGA5: Molecular Evolutionary Genetics Analysis Using Maximum Likelihood, Evolutionary Distance, and Maximum Parsimony Methods. Mol. Biol. Evol. 2011, 28, $2731-2739$. [CrossRef]

34. Bailey, T.L.; Williams, N.; Misleh, C.; Li, W.W. MEME: Discovering and analyzing DNA and protein sequence motifs. Nucleic Acids Res. 2006, 34, W369e73. [CrossRef] 
35. Eisenhaber, B.; Schneider, G.; Wildpaner, M.; Eisenhaber, F. A sensitive predictor for potential GPI lipid modification sites in fungal protein sequences and its application to genome-wide studies for Aspergillus nidulans, Candida albicans, Neurospora crassa, Saccharomyces cerevisiae, and Schizosaccharomyces pombe. J. Mol. Biol. 2004, 337, 243-253. [CrossRef]

36. Brown, N.A.; Antoniw, J.; Hammond-Kosack, K.E. The predicted secretome of the plant pathogenic fungus Fusarium graminearum: A refined comparative analysis. PLoS ONE 2012, 7, e33731. [CrossRef]

37. Sperschneider, J.; Gardiner, D.M.; Dodds, P.N.; Tini, F.; Covarelli, L.; Singh, K.B.; Manners, J.M.; Taylor, J.M. EffectorP: Predicting fungal effector proteins from secretomes using machine learning. New Phytol. 2016, 210, 743-761. [CrossRef]

38. Krüger, A.T.; Engel, J.; Buettner, F.F.; Routier, F.H. Aspergillus fumigatus Cap59-like protein A is involved in $\alpha 1,3-$ mannosylation of GPI-anchors. Glycobiology 2016, 26, 30-38.

39. Lo Presti, L.; Lanver, D.; Schweizer, G.; Tanaka, S.; Liang, L.; Tollot, M.; Zuccaro, A.; Reissmann, S.; Kahmann, R. Fungal effectors and plant susceptibility. Annu. Rev. Plant Biol. 2015, 66, 513-545. [CrossRef]

40. Nasser, L.; Weissman, Z.; Pinsky, M.; Amartely, H.; Dvir, H.; Kornitzer, D. Structural basis of haem-iron acquisition by fungal pathogens. Nat. Microbiol. 2016, 1, 16156. [CrossRef]

41. Gong, A.D.; Jing, Z.Y.; Zhang, K.; Tan, Q.Q.; Wang, G.L.; Liu, W.D. Bioinformatic analysis and functional characterization of the CFEM proteins in maize anthracnose fungus Colletotrichum graminicola. J. Integr. Agric. 2020, 19, 541-550. [CrossRef]

42. Moukadiri, I.; Armero, J.; Abad, A.; Sentandreu, R.; Zueco, J. Identification of a mannoprotein present in the inner layer of the cell wall of Saccharomyces cerevisiae. J. Bacteriol. 1997, 179, 2154-2162. [CrossRef]

43. Sabnam, N.; Roy-Barman, S. WISH, a novel CFEM GPCR is indispensable for surface sensing, asexual and pathogenic differentiation in rice blast fungus. Fungal Genet. Biol. 2017, 105, 37-51. [CrossRef]

44. Yang, F.; Jensen, J.D.; Svensson, B.; Jorgensen, H.J.L.; Collinge, D.B.; Finnie, C. Secretomics identifies Fusarium graminearum proteins involved in the interaction with barley and wheat. Mol. Plant Pathol. 2012, 13, 445-453. [CrossRef]

45. Nombela, C.; Gil, C.; Chaffin, W.L. Non-conventional protein secretion in yeast. Trends Microbiol. 2006, 14, 15-21. [CrossRef]

46. Eisenhaber, B.; Bork, P.; Eisenhaber, F. Post-translational GPI lipid anchor modification of proteins in kingdoms of life: Analysis of protein sequence data from complete genomes. Protein Eng. Des. Sel. 2001, 14, 17-25. [CrossRef] 